\title{
Controlling and enhancing synchronisation through adaptive phase lags
}

\author{
Alexander C. Kalloniatis \\ Joint and Operations Analysis Division, \\ Defence Science and Technology Group, \\ Canberra, Australia 2600. \\ Markus Brede \\ Complexity, Agents and Interactions, \\ Department of Electronics and Computer Science, \\ University of Southampton, United Kingdom
}

(Dated: February 18, 2019)

\begin{abstract}
We compare two methods for controlling synchronisation in the Kuramoto model on an undirected network. The first is by driving selected oscillators at a desired frequency by coupling to an external driver, and the second is by including adaptive lags - or dynamical frustrations - within the Kuramoto interactions, with the lags evolving according to a dynamics as a function of the reference frequency with an associated time-constant. Performing numerical simulations with random regular graphs, we find that above a certain connectivity driving via adaptive lags allows for stronger alignment to the external frequency at lower value of the time-constant compared to the corresponding coupling strength for the externally driven model. Numerical results are backed up by equilibrium analysis based on a fixed point ansatz for frequency synchronised clusters where we solve the spectrum of the associated Jacobian matrix. We find that at low connectivity the external driving mechanism is successful down to lower densities of controlled oscillators where the adaptive lag approach is Lyapunov unstable at all densities. As connectivity increases, however, the adaptive lag mechanism shows stability over similar ranges of density to the external driving and proves superior in terms of tighter splays of oscillators. In particular, the threshold for instability for the adaptive lag model shows robustness against variations in the associated time constant down to lower densities of controlled oscillators. A simple intuitive model emerges based on the interaction between splayed clusters close to a critical point.
\end{abstract}

PACS numbers: 05.45.Xt, 05.70.Ln, 89.75.Fb, 89.75.Hc

\section{INTRODUCTION}

The ability of networks of coupled entities to achieve synchronised dynamics is fundamental to many scientific applications, from physical, chemical, biological and even social or human organisational systems. To this end, the Kuramoto model [1] on a network, defined by

$$
\dot{\theta}_{i}=\omega_{i}+\frac{K}{N} \sum_{j=1}^{N} A_{i j} \sin \left(\theta_{j}-\theta_{i}\right),
$$

integrates a number of simple features such as structure, heterogeneity and cyclic dynamics while displaying quite rich behaviours (see [2-5] for recent reviews). Here $\theta_{i}$ is a time-dependent phase angle at node $i$ of a network of $N$ nodes of a graph $\mathcal{G}$ given by the adjacency matrix $A_{i j}$, with $\omega_{i}$ the native frequencies drawn from some statistical distribution. The model is well known to display a critical transition for the complete graph for $N \rightarrow \infty$ at some coupling $K$ at which a proportion of oscillators spontaneously synchronise to a frequency (for undirected graphs) that is the mean of the native frequency ensemble $\bar{\omega}$; the normalised coupling may be absorbed into a constant $\sigma \equiv \frac{K}{N}$. Many papers have explored this syncronisation for general networks, and the role of topology in influencing this effect [6-11] or the inter-relationship between topology and the frequency allocation on the network [12]. More recently attention has turned to control problems in this context. The standard approach to control on a network is to attach external controllers to the nodes on which is defined a linear dynamical system, and pose the analogous question to the Kalman filter [13]: is the network controllable in the sense of any final state being reachable from any initial state? In synchronisation problems this is more narrowly defined: can the system be controlled to reach synchrony from any random initial condition? Implicit in this objective is synchrony to the mean of the frequency ensemble. In this paper we shall explore a mechanism that improves synchronisation, namely at lower 'cost' - in terms of coupling strength and to frequencies other than the mean of the ensemble of native frequencies. Such a formulation of the control problem is relevant in a range of contexts of engineered systems where coordination is a requirement, from vehicle motion [14], and power systems [15], to robot [16] and human-robot interactions [17].

The classical work on external controllability on networks is that of Liu, Slotine and Barabasi [18] who adapt the Kalman filter approach. As inferred, here the objective is to guarantee that any final state of a vector of variables $X_{i}$ is achievable from any initial input using linear control. More recently, the work has also been generalised to some forms of non-linear control by Arsiwalla, Barzel and Barabasi [19]. Within the linear 
model, problems of minimum number of nodes and minimum cost have been explored by Li et al. [20]. This has been extended to multiplex networks by Posfai et al. [21]. Within synchronisation problems, the final state is more prescribed, namely frequency- or phase-synchrony. Hamiltonian control for the Kuramoto model has been explored by Gjata et al [22], where the Kuramoto model can be written in Hamilton form using action-angles variables [23-25]. This method also works in the regime of partial synchronisation where perturbations then allow the fully synchronised state to be achieved. Closer in spirit to our approach, an adaptive model of control has been explored by Eom, Boccaletti and Caldarelli [26], but where the network itself evolves through a fitness model to improve synchronisation. An alternative approach considers an adaptive coupling [27]. In all of these cases the adaptation improves synchronisation to the mean frequency. Are different collective frequencies possible? May control facilitate this?

One way of controlling to a different frequency is that of pinning control, where one adds to Eq. (1) an additional term, say, $u_{i}$ that assumes some functional form and is non-zero only for particular nodes, a subset that seeks to pin the entire system to a specified behaviour. For more general Laplacian coupled systems, such as used in the Master Stability approach of [39], this has been advanced by [28] and [29]. In the Kuramoto model, variations of this have been explored by [30] and [31]. All these approaches use Lyapunov stability to determine criteria for achieving the desired controllability.

Our paper takes its cue from the lesser-known Kuramoto-Sakaguchi model which introduces a phase lag or frustration $\lambda_{i}$

$$
\dot{\theta}_{i}=\omega_{i}+\frac{K}{N} \sum_{j=1}^{N} A_{i j} \sin \left(\theta_{j}-\theta_{i}+\lambda_{i}\right),
$$

This model is known for the property that the system does not synchronise to the mean of the ensemble by virtue of the absence now of the antisymmetry of the interaction function. In [32], a version of this model with pinning terms, such as described above, was examined for controllability. In [33], it was observed that lags alone may be used to control the system to a predefined frequency for the complete network case. In [34], a similar mechanism was used for sparser graphs to enable frequencies to be selected in order to provide for perfect synchronisation. In our previous paper [35] we showed how a form of codynamics, namely adaptive lags, where the $\lambda_{i}$ become functions of time with their own evolution equation, can improve synchronisation for sparse networks - indeed, achieve perfect phase synchronisation. This arises essentially because the phase shifts achieve an equilibrium configuration that overcomes the barrier posed by the non-uniform native frequencies for perfect synchronisation $\theta_{i}=\theta_{j}$. However, we observed that the actual equilibrium state the system may end up in is unreliable for arbitrary initial conditions in $[-\pi, \pi]$, so that the system from a random initial condition was not guaranteed to synchronise to the selected external frequency.

In this paper we modify our adaptive lag mechanism of [35] to show how it can become a control model in the sense of guaranteeing synchronisation to a specified external frequency. However, we compare the adaptive lag model to the pinning control approach cited earlier, which, due to its precedence we refer to as the reference model. We find, in fact, that the adaptive model is more efficient in terms of required controlled nodes at achieving the desired frequency and with better synchronisation for networks above a minimum level of connectivity. In this paper we focus on random regular networks, where there is enough heterogeneity but we are able to isolate systematically the role of connectivity. In numerical examples, we consider networks of size $N=40$ where long transients do not inhibit elucidation of the underlying mechanisms, however we have run the full numerical computations for larger scale systems, for $N \sim 1000$ and find qualitatively similar results.

The paper is structured as follows. First we outline the adaptive and reference models explicitly. We then show a range of numerical simulations of the model for a network of $N=40$ nodes, and identify the different phases of the system and for different connectivities for random regular graphs. We then conduct a fixed point analysis to examine how stability properties relate to the regimes we observe numerically. Important for this will be an ansatz based on two clusters of nodes on the network - those subject to control and those not. We then consider a range of connectivities in the spectral analysis and demonstrate results consistent with the full numerical computations. We examine the coupling dependence of the two models within the linearised approach. We finally summarise and conclude with prospects of the applicability of such a mechanism.

\section{DEFINING THE MODELS: ADAPTIVE LAGS VS EXTERNAL CONTROL}

We compare two models where Kuramoto sychronisation is controlled to some external driving frequency $\Omega$. The first we may refer to as a reference model:

$$
\begin{aligned}
\dot{\theta}= & \omega_{i}+\sigma \sum_{j} A_{i j} \sin \left(\theta_{j}-\theta_{i}\right) \\
& +\eta b_{i} \sin \left(\Omega t-\theta_{i}\right)
\end{aligned}
$$

Here, an oscillator $\theta_{i}$ couples to an external driver when $b_{i}=1$ or is left to itself to synchronise according to its adjacent partners when $b_{i}=0$. The driver strength is given by $\eta$.

An alternate model is one where time-dependent lags $\lambda_{i}(t)$ are introduced but which co-evolve with the $\theta_{i}$ :

$$
\begin{aligned}
& \dot{\theta}_{i}=\omega_{i}+\sigma \sum_{j} A_{i j} \sin \left(\theta_{j}-\theta_{i}+\lambda_{i}\right) \\
& \dot{\lambda}_{i}=\tau b_{i} \sin \left(\Omega t-\theta_{i}\right) .
\end{aligned}
$$


Here again, when $b_{i}=1$ the lags co-evolve and when $b_{i}=0$ they are frozen in which case we assign initial condition $\lambda_{i}(0)=0$, or simply $\lambda_{i}(0)=b_{i} \lambda$ with $\lambda \in(-\pi, \pi)$ drawn at random from a uniform distribution. The phases $\theta_{i}$ corresponding to nodes with $b_{i}=0$ then evolve according to their own local synchronisation interactions on the network. The constant $\tau$ here represents the time constant associated wth the dynamical lags. One motivation for the second model is our earlier work on adaptive lag dynamics $\dot{\lambda}=\tau \sum_{j} A_{i j} \sin \left(\theta_{j}-\theta_{i}\right)$ where we found this could enhance synchronisation compared to the ordinary Kuramoto dynamics while failing to guarantee the external collective frequency to which this synchronisation occurs. The present version elevates this interaction to a control model with explicit reference to an external driving frequency $\Omega$.

We wish to compare the behaviours of these models in terms of driving phases to the external frequency, $\theta_{i} \approx \Omega t$, as functions of the various strengths $\eta, \tau$ as the density of control nodes, $\rho=\sum_{i} b_{i} / N$ varies between 0 and 1.

\section{BEHAVIOURS FOR RANDOM REGULAR NETWORKS: NUMERICAL SOLUTIONS}

We begin by showing the results of numerical integration of the equations of motion for the two models. Using a fourth order Runge-Kutta method with step size $\Delta t=0.1$ we integrate out to $t=5 \times 10^{5}$ time steps for $\sigma=1$. Simulations are carried out for random regular networks of degree $k$, a deliberate choice to reduce effects of network heterogeneity in this initial investigation. We explore the choices $k=6,8,12$ in the following. Native frequencies $\omega_{i}$ are randomly drawn from the uniform distribution $[-1,1]$ so that for each instance the average frequency $\bar{\omega} \approx 0$. We choose $\Omega=2.5$ - a controlling frequency clearly outside the interval for the native frequencies. We scan across the remaining two parameters for the two models: $(\rho, \eta)$ for the reference model Eq. (3), and $(\rho, \tau)$ for the adaptive lags Eq. (4). The degree of synchronisation is measured by Kuramoto's order parameter

$$
r=\left\langle\frac{1}{N}\left|\sum_{j} e^{i \theta_{j}}\right|\right\rangle
$$

where brackets indicate averages over time (after discarding a transient), and where $r=1$ represents full synchronisation, and $r=0$ pure incoherence. We also measure the closeness of the average instantaneous frequency to the external frequency $\Omega$ via

$$
\left.\Delta=\left\langle\frac{1}{N} \sum_{i}\right| \dot{\theta}_{i}-\Omega\right)|\rangle,
$$

where $\Delta=0$ means success in driving to the external frequency.

Numerical experiments show that given configurations either achieve very good synchronisation to the external
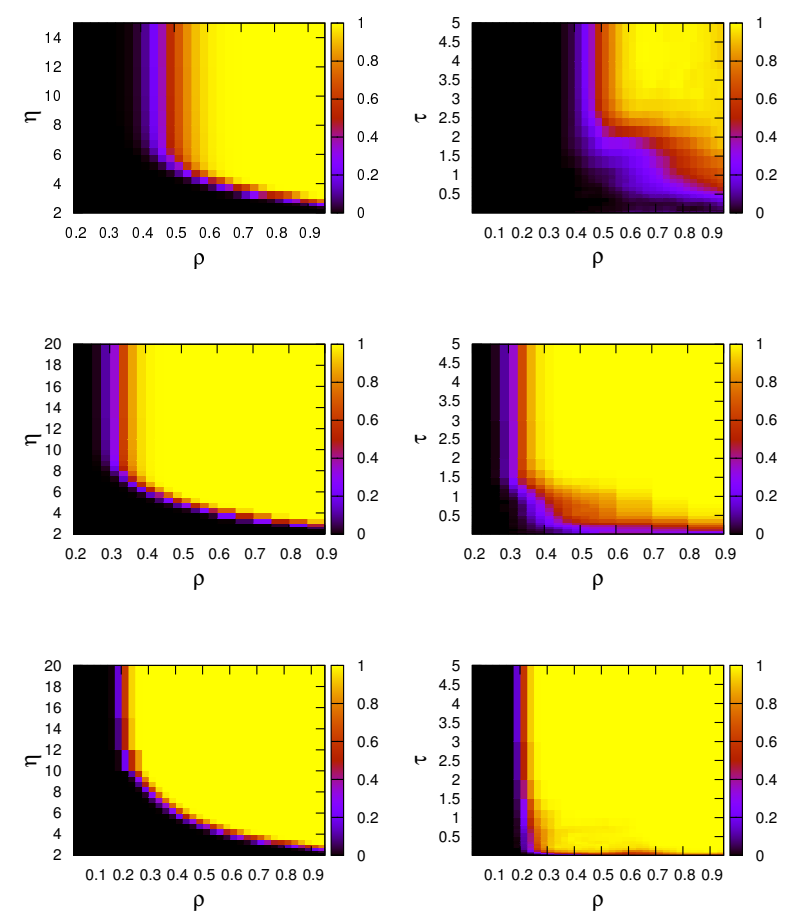

FIG. 1. Phase diagrams for random regular graph of $N=40$, showing the fraction of 100 instances achieving full synchronisation to the frequency $\Omega$ across the parameter space of $\rho$ vs $\tau$ for the adaptive lag model (left) and $\rho$ vs $\eta$ for the externally driven reference model (right), with different connectivities $k=6,8,12$ respectively top, middle and bottom rows.

frequency $\left(\Delta<10^{-4}\right)$ or fail to synchronise. Accordingly, for given densities of controlled oscillators $\rho$ and given parameters $\tau$ or $\eta$ we measure the fraction of configurations that achieved synchronisation to the external frequency. In Fig. 1 we show density plots for the fraction of configurations achieving full synchronisation to the frequency $\Omega$ : brighter regions indicate that systems achieve full synchrony, while black indicates regions in which configurations fail to synchronise. The left column shows the reference model for increasing $k$ down the column, and the right shows the adaptive lags model. We note that for low values of $\tau$ transients become very long - which we shall explain later - and so for $\tau<0.5$ one must integrate up to very large times to establish whether an instance has synchronised. Hence numerical results in this region have to be seen as a lower bound for the number of synchronised configurations. For the same reason we do not go to lower values of $k$, such as $k=4$ where the transients become inordinately long and numerical instabilities arise. The three choices of $k$ serve to make the point of where the various models are effective.

First we observe in Fig. 1 a minimum density of controlled oscillators $\rho$ for synchronisation to occur and this varies with $k$. For the reference model, this goes from $\rho_{c} \approx 0.45$ for lower $k$ down to $\rho_{c} \approx 0.2$ for the higher 
$k$. A similar behaviour occurs for the adaptive lag model after a poorer performance at the lowest $k$. Thus the adaptive lag model does not perform as efficiently as the reference model for low connectivity. More interestingly, we see that the adaptive lag model allows for synchronisation to the driving frequency even at very small values of $\tau$, whereas the reference model clearly requires substantial coupling strength $\eta$. The contrast with the reference model is unambiguous: below $\eta=1$ systems do not synchronise at any $\rho$, while in the lag-controlled model at least some synchronised configurations are observed for any choice of $\tau$. Close to $100 \%$ synchronised configurations are achieved for much lower values of $\tau \geq 1$ for the lag-controlled case as compared to $\eta \geq 8$ in the reference model.

To summarise these behaviours then, we see that the adaptive lag model is effective down to lower coupling strengths than the reference model, though only taking effect above a certain minimum connectivity, but then with similar critical densities of controlled nodes. The adaptive lad model also exhibits long transients to equilibrium. In the following we seek to understand these behaviours more analytically using fixed point analysis.

\section{ANALYTIC RESULTS THROUGH LINEARISATION: TWO CLUSTER ANSATZ AND STABILITY}

\section{A. Ansatz for the adaptive lag model}

Prompted by the observation in the previous section that for general $\rho$ synchronisation to the external frequency, though very high, is never quite perfect, we perform an equilibrium analysis allowing for two clusters in the fixed point ansatz: one consisting of the oscillators that phase synchronise alongside controlling phase lags $\left(b_{i}=1\right)$, identified by nodes in a sub-graph, which we denote $i \in \mathcal{G}_{1}$, and the other those nodes without lags $\left(b_{i}=0\right), i \in \mathcal{G}_{2}=\mathcal{G}-\mathcal{G}_{1}$. So the ansatz reads

$$
\begin{aligned}
\theta_{i}(t) & =\Omega t+\vartheta_{i}(t), i \in \mathcal{G}_{1} \\
\theta_{i}(t) & =\Omega t+\alpha_{i}+\varphi_{i}(t), i \in \mathcal{G}_{2} \\
\lambda_{i}(t) & =\mu_{i}+\chi_{i}(t)
\end{aligned}
$$

with $\vartheta_{i}, \varphi_{i}$ and $\chi_{i}$ considered as small fluctuations.

One of us has used ansaetze such as this in the study of a multi-network generalisation of the Kuramoto model $[36,37]$, where we allow for two or three relatively shifted but internally phase synchronised clusters. Within such tight constraints, a great deal of analytical tractability is obtained. Here we relax the requirement of exact phase synchronisation within the second cluster. In this respect we allow the cluster to display an arbitrary degree of 'splay', $\alpha_{i} \neq \alpha_{j}$, whose consequences will be seen below.

Expanding the equations of motion Eqs. (4) we obtain

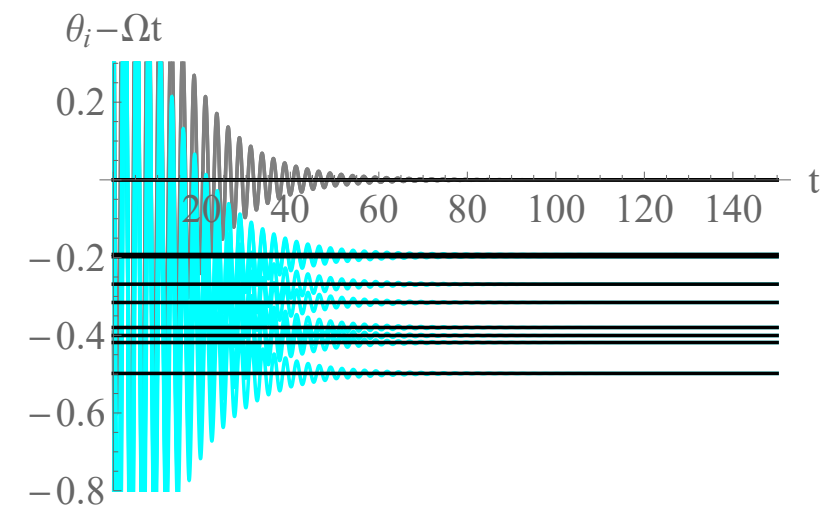

FIG. 2. Example of clustering for $N=40, \rho=0.8$ where grey are the controlled $\theta_{i}-\Omega t$, cyan are the uncontrolled $\theta_{i}-\Omega t$ and black are the $\alpha_{i}$ derived from Eq. (11).

$$
\begin{aligned}
\dot{\vartheta}_{i}= & \omega_{i}-\Omega+\sigma k_{i}^{(1)} \sin \mu_{i}+\sigma k_{i}^{(2)} \sin \left(\alpha_{i}+\mu_{i}\right) \\
& +\mathcal{O}_{i}^{(1)}(\vartheta, \varphi, \chi) \\
\dot{\varphi}_{i}= & \omega_{i}-\Omega-\sigma k_{i}^{(1)} \sin \alpha_{i}+\sigma \sum_{j \in \mathcal{G}_{2}} A_{i j} \sin \left(\alpha_{j}-\alpha_{i}\right) \\
& +\mathcal{O}_{i}^{(2)}(\vartheta, \varphi, \chi) \\
\dot{\chi}_{i}= & -\tau \varphi_{i}
\end{aligned}
$$

where $k_{i}^{(a)}$ is the degree of node $i$ with respect to nodes in the sub-graph $\mathcal{G}_{a}$, and $\mathcal{O}_{i}^{(a)}$ are linear in the fluctuations, to be given explicitly below. Note here that we may form the fluctuations into a super-vector $v=(\vartheta, \varphi, \chi)$ so that the overall linearised system takes the form

$$
\dot{v}=W-\Lambda v
$$

with $\Lambda$ the Jacobian which we give explicitly in the next section. Thus steady-state solutions, if the system is stable, are $v^{*}=\Lambda^{-1} W$ after removal of any zero-modes (or alternately, using the pseudo-inverse).

Requiring that the $\dot{v}$ vanish, so that all the constant parts are in the parameters $\mu_{i}, \alpha_{i}$, gives:

$$
\begin{aligned}
\omega_{i}-\Omega+\sigma k_{i}^{(1)} \sin \mu_{i}+\sigma k_{i}^{(2)} \sin \left(\alpha_{i}+\mu_{i}\right) & =0 \\
i \in \mathcal{G}_{1} & \\
\omega_{i}-\Omega-\sigma k_{i}^{(1)} \sin \alpha_{i}+\sigma \sum_{j \in \mathcal{G}_{2}} A_{i j} \sin \left(\alpha_{j}-\alpha_{i}\right) & =0 \\
i \in \mathcal{G}_{2} . &
\end{aligned}
$$

Eqs. (10,11) now define the fixed point manifold. The obvious procedure is to use the second of these, Eq. (11), which is $\mu_{i}$ independent, to solve for $\alpha_{i}$ first and then use these in the first Eq. (10) to solve for the $\mu_{i}$. The $\alpha_{i}$ equation may be simplified by assuming $\alpha_{i}-\alpha_{j}$ to be small allowing the $\sin \left(\alpha_{j}-\alpha_{i}\right)$ term to be re-expressed in terms of the graph Laplacian. Unfortunately, there is no analytical solution for this, so we do not pursue this option. However, the first equation may be solved for 


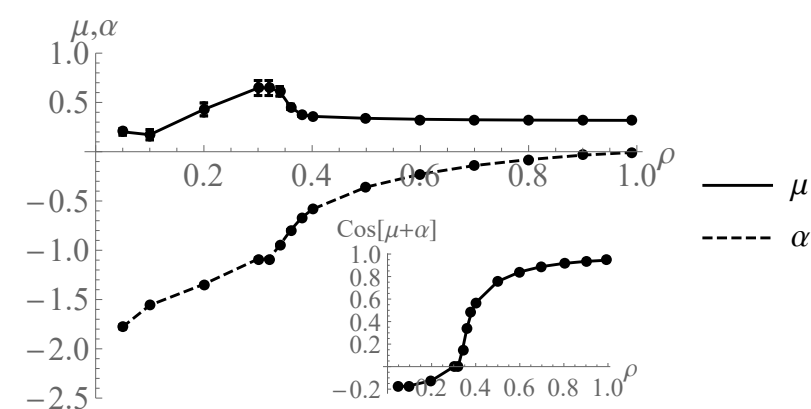

FIG. 3. Plots of $\alpha=\frac{1}{N} \sum_{i} \alpha_{i}$ (dashed) and $\mu=\frac{1}{N} \sum_{i} \mu_{i}$ (solid) from Eqs. $(10,11)$ in the adaptive lag model for $N=40$ random regular $k_{i}=8$ with $\sigma=\tau=1, \Omega=2.5$, averaged over 300 instances, for different values of $\rho$. Note that these are independent of $\tau$. Inset: plot of $\frac{1}{N} \sum_{i} \cos \left(\mu_{i}+\alpha_{i}\right)$ averaged over the same instances. Error bars here and in subsequent plots represent the standard error.

$\sin \mu_{i}$ :

$$
\begin{aligned}
\sin \mu_{i}= & \left(-\left(\omega_{i}-\Omega\right)\left(k_{i}^{(1)}+k_{i}^{(2)} \cos \alpha_{i}\right)\right. \\
& \pm k_{i}^{(2)}\left|\sin \alpha_{i}\right|\left[\sigma^{2}\left(k_{i}^{(1)}\right)^{2}+2 k_{i}^{(1)} k_{i}^{(2)} \cos \alpha_{i}\right. \\
& \left.\left.+\left(k_{i}^{(2)}\right)^{2}-\left(\omega_{i}-\Omega\right)^{2}\right]^{\frac{1}{2}}\right) / \\
& \left.\left(\sigma\left(k_{i}^{(1)}\right)^{2}+2 k_{i}^{(1)} k_{i}^{(2)} \cos \alpha_{i}+\left(k_{i}^{(2)}\right)^{2}\right)\right) .
\end{aligned}
$$

If we subject all nodes to control so that $\alpha_{i}=0$, in which case $k_{i}=k_{i}^{(1)}$, then $\sin \mu_{i}=-\frac{\omega_{i}-\Omega}{\sigma k_{i}}$. Such a result for the equilibrium phase lags arises in [35] where a different model was used for the adaptive mechanism.

To illustrate clustering as one tight group and another splayed, we show in Fig. 2 a run of a random regular graph for $N=40$ with $\rho=0.8, \sigma=\tau=1$, and, again, $\Omega=2.5$. We have solved the system numerically in this case using Mathematica's NDSolve function. The plot shows the behaviour of $\theta_{i}-\Omega t$ for both controlled (gray) and uncontrolled (cyan) nodes. We see that the 32 controlled components, with $b_{i}=1$, give $\theta_{i}=\Omega t$ demonstrating their perfect phase synchronisation. The remaining eight uncontrolled components show deviations from $\Omega t$ that are each slightly different from each other, and rapidly converge to the $\alpha_{i}$ as derived from Eq. (11), indicated in black.

Extending this now to better understand the fixed point, we examine the cases of $k=4,5,8,12,14$, outside the range of the full simulation solutions of Sect.III. We use values $\tau=0.2,2,20,200$ for the adaptive lag model and compute $\alpha_{i}$ for $i \in \mathcal{G}_{2}$ from Eq. (11), followed by $\mu_{i}$ for $i \in \mathcal{G}_{1}$ from Eq. (10). We show an example of this result for the case $k=8$ in Fig. 3. For other values of $k$ the dependence on $\rho$ is similar: for smaller $k$ the dependence of $\cos (\mu+\alpha)$ is more linear in $\rho$. The $\mu_{i}, \alpha_{i}$ are evidently $\tau$ independent from their defining equations.

But the key obsrvation from this, which will inform the intuitive picture in the sequel, is that as $\rho$ decreases the $\mu$ stay nearly constant while $\alpha$ increases in magnitude until $\rho=0.4$, at which point even the equilibrium lag values $\mu$ see a slight increase around $\rho=0.3$. We also present, as inset, the behaviour of $\cos (\mu+\alpha)$ which shows a sign change at the point where $\mu$ becomes sensitive to the value of $\rho$; in antifcipation of the spectra, we note that the combination $\cos (\mu+\alpha)$ recurs through the terms of the super-Laplacian.

\section{B. Stability analysis of adaptive lags}

We now examine the fluctuations around the fixed point in Eqs. $(10,11)$. In components, the Jacobian $\Lambda^{\text {AdLg }}$ is:

$$
\Lambda_{i j}^{\mathrm{AdLg}}=\left(\begin{array}{ccc}
\Lambda_{i j}^{\vartheta \vartheta} & \Lambda_{i j}^{\vartheta \varphi} & \Lambda_{i j}^{\vartheta \chi} \\
\Lambda_{i j}^{\varphi \vartheta} & \Lambda_{i j}^{\varphi \varphi} & 0 \\
\tau \delta_{i j} & 0 & 0
\end{array}\right)
$$

where

$$
\begin{aligned}
\Lambda_{i j}^{\vartheta \vartheta}= & \sigma \cos \mu_{i} L_{i j}^{(11)} \\
& +\sigma \sum_{j^{\prime} \in \mathcal{G}_{2}} \cos \left(\alpha_{j^{\prime}}+\mu_{i}\right) A_{i j^{\prime}}^{(12)} \delta_{i j}, \\
\Lambda_{i j}^{\vartheta \varphi}= & -\sigma A_{i j}^{(12)} \cos \left(\alpha_{j}+\mu_{i}\right), \\
\Lambda_{i j}^{\vartheta \chi}= & -\sigma D_{i j}^{(11)} \cos \mu_{i} \\
& -\sigma \sum_{j^{\prime} \in \mathcal{G}_{2}} A_{i j^{\prime}}^{(12)} \cos \left(\mu_{i}+\alpha_{j^{\prime}}\right) \delta_{i j}, \\
\Lambda_{i j}^{\varphi \vartheta}= & -\sigma A_{i j}^{(21)} \cos \alpha_{i}, \\
\Lambda_{i j}^{\varphi \varphi}= & \sigma L_{i j}^{(22)} \cos \left(\alpha_{i}-\alpha_{j}\right)+\sigma D_{i j}^{(21)} \cos \alpha_{i},
\end{aligned}
$$

where $D_{i j}^{(a b)}$ represents the diagonal matrix of degrees of the nodes of sub-graph $\mathcal{G}_{a}$ connected to $\mathcal{G}_{b}$, and $L_{i j}^{(a b)}=D_{i j}^{(a b)}-A_{i j}^{(a b)}$ represents the corresponding Laplacian. The appearance of Laplacians of the various subgraphs within these components means we will occasionally refer to $\Lambda^{\text {AdLg }}$ as a 'super-Laplacian'.

The spectrum of the ordinary ('combinatorial') graph Laplacian [38] is generally known to play an important role in understanding coupled dynamical systems on networks [39]. While not quite as straightforward for the Kuramoto model, the lowest eigenvalue, known as the Fiedler [40], indicates the slowest transient of the system [41] close to synchronisation. But the entire spectrum leaves an imprint in the dynamics across a range of coupling, even quite far from synchronisation [42, 43]. Much is known about the spectrum of the graph Laplacian for the classical graphs [44], which enables us to infer some properties of the spectrum of $\Lambda^{\mathrm{AdLg}}$.

In the absence of clustering, $\alpha_{i}=0 \forall i$, the superLaplacian $\Lambda_{i j}^{\mathrm{AdLg}}$ involves factors of $\cos \mu_{i}$, a form quite close to that encountered in our previous work [35], and also not dissimilar from that found from a stability analysis of the ordinary Kuramoto-Sakaguchi model, Eq. (2). 
We know there that $\cos \mu_{i}>0$ provides a necessary condition for stability. We thus can expect thresholds for instability when $\alpha_{i} \neq 0$. Moreover, $\Lambda^{\mathrm{AdLg}}$ here is not symmetric, so we expect a complex valued spectrum.

We defer momentarily exploring these results for specific cases.

\section{Ansatz and stability analysis for reference model}

We now perform a similar analysis for the reference control model. With the details given previously we may be a little circumspect here. Firstly, there are no explicit lags here, so in view of the possibility that perfect phase synchronisation might not be achievable we include $\mu_{i}$ in the ansatz

$$
\begin{aligned}
& \theta_{i}(t)=\Omega t+\mu_{i}+\vartheta_{i}(t), i \in \mathcal{G}_{1} \\
& \theta_{i}(t)=\Omega t+\alpha_{i}+\varphi_{i}(t), i \in \mathcal{G}_{2} .
\end{aligned}
$$

This gives for the fixed point (ignoring fluctuations after inserting the ansatz in the defining equations)

$$
\begin{gathered}
\omega_{i}-\Omega+\sigma \sum_{j \in \mathcal{G}_{1}} A_{i j} \sin \left(\mu_{j}-\mu_{i}\right) \\
+\sigma \sum_{j \in \mathcal{G}_{2}} A_{i j} \sin \left(\alpha_{j}-\mu_{i}\right)-\eta \sin \mu_{i}=0, \\
i \in \mathcal{G}_{1}, \\
\omega_{i}-\Omega+\sigma \sum_{j \in \mathcal{G}_{1}} A_{i j} \sin \left(\mu_{j}-\alpha_{i}\right) \\
+\sigma \sum_{j \in \mathcal{G}_{2}} A_{i j} \sin \left(\alpha_{j}-\alpha_{i}\right)=0, \\
i \in \mathcal{G}_{2} .
\end{gathered}
$$

To visualise the equilibrium values of $\alpha_{i}$ and $\mu_{i}$ from Eqs. $(19,20)$ in the reference model we plot the results for $k=8$ shown in Fig. 4; other values show similar behaviour. We use $\eta=1,5,10,100$ here. Note that with the explicit $\eta$-dependence in Eq. (19) the figure is somewhat more complex. At one extreme, $\eta=100$, the $\alpha_{i}$ increasing with decreasing $\rho$ while $\mu_{i}$ are vanishingly small across the range of fraction of the controlled oscillators. Evidently, with such strong driving the controlled oscillators achieve very small phase lags. As $\rho$ is reduced, the number of uncontrolled oscillators increases so that a splay develops in this group, with $\alpha_{i}$ increasing in magnitude. As $\eta$ decreases values of $\mu$ become non-zero but show insensitivity to the value of $\rho$ down to some critical value. But the splay, as seen in $\alpha$, increases as the number of controlled oscillators decreases. Importantly, very high coupling $\eta$ to the driving frequency is required to achieve 'perfect phase synchronisation' $\mu_{i} \approx 0$. At the other extreme, $\eta=1$, the driving is so weak that the underlying Kuramoto interaction, with coupling $\sigma$, causes even the driven oscillators to be splayed. As $\rho$ decreases, fewer oscillators are driven, so more may participate in the Kuramoto interaction. To say more beyond the crossover point requires completing the stability analysis.
The Jacobian is now a two-by-two block form

$$
\Lambda_{i j}^{\operatorname{Ref}}=\left(\begin{array}{cc}
\Lambda_{i j}^{\vartheta \vartheta} & \Lambda_{i j}^{\vartheta \varphi} \\
\Lambda_{i j}^{\varphi \vartheta} & \Lambda_{i j}^{\varphi \varphi}
\end{array}\right),
$$

where the individual entries are:

$$
\begin{aligned}
\Lambda_{i j}^{\vartheta \vartheta}= & \sigma L_{i j}^{(11)} \cos \left(\mu_{i}-\mu_{j}\right) \\
& +\sigma \sum_{j^{\prime} \in \mathcal{G}_{2}} A_{i j^{\prime}}^{(12)} \cos \left(\alpha_{j^{\prime}}-\mu_{i}\right) \delta_{i j} \\
& +\eta \cos \mu_{i}, \\
\Lambda_{i j}^{\vartheta \varphi}= & -\sigma A_{i j}^{(12)} \cos \left(\alpha_{j}-\mu_{i}\right), \\
\Lambda_{i j}^{\varphi \vartheta}= & -\sigma A_{i j}^{(21)} \cos \left(\mu_{j}-\alpha_{i}\right), \\
\Lambda_{i j}^{\varphi \varphi}= & \sigma L_{i j}^{(22)} \cos \left(\alpha_{i}-\alpha_{j}\right) \\
& +\sigma \sum_{j^{\prime} \in \mathcal{G}_{1}} A_{i j^{\prime}}^{(21)} \cos \left(\mu_{j^{\prime}}-\alpha_{i}\right) \delta_{i j} .
\end{aligned}
$$

These entries are more standard weighted Laplacians, apart from the shift $\eta \cos \mu_{i}$ whose sign is contingent on the $\mu_{i}$. Overall $\Lambda^{\text {Ref }}$ is symmetric, and therefore we may expect a purely real spectrum but with a shift in the lowest eigenvalue according to the values of $\mu_{i}$. Alternately put, for $\eta=0, \Lambda^{\text {Ref }}$ has zero row and column sums and therefore has a spectrum bounded below by a zero eigenvalue.

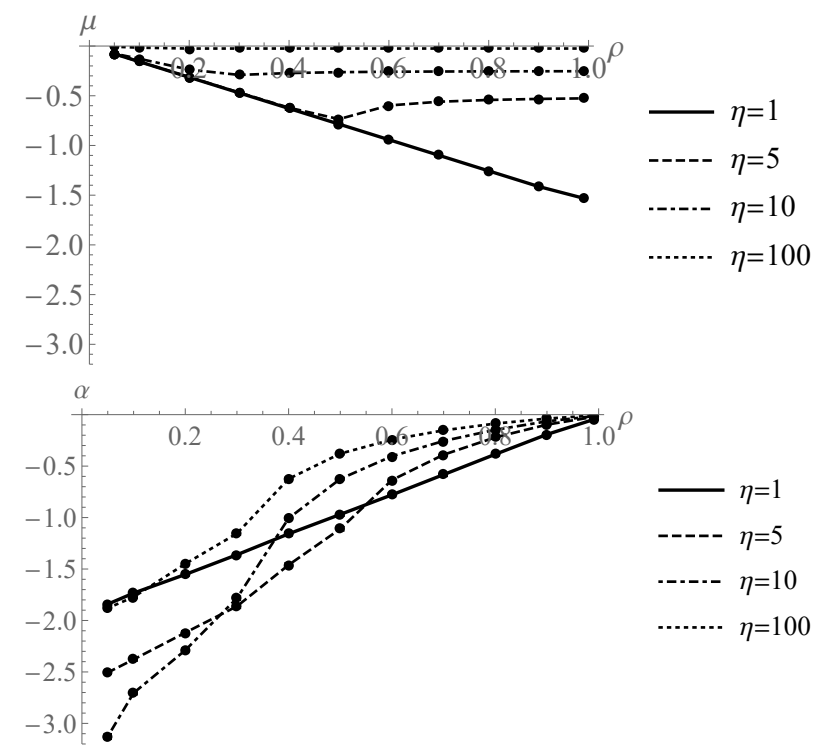

FIG. 4. Plots of $\mu=\frac{1}{N} \sum_{i} \mu_{i}$ (top) and $\alpha=\frac{1}{N} \sum_{i} \alpha_{i}$ (bottom) in the externally driven model from Eqs. $(20,19)$ for $N=40$ random regular $k_{i}=8$ with $\sigma=\tau=1, \Omega=2.5$, averaged over 300 instances, as a function of $\rho$ for different values of $\eta$. 


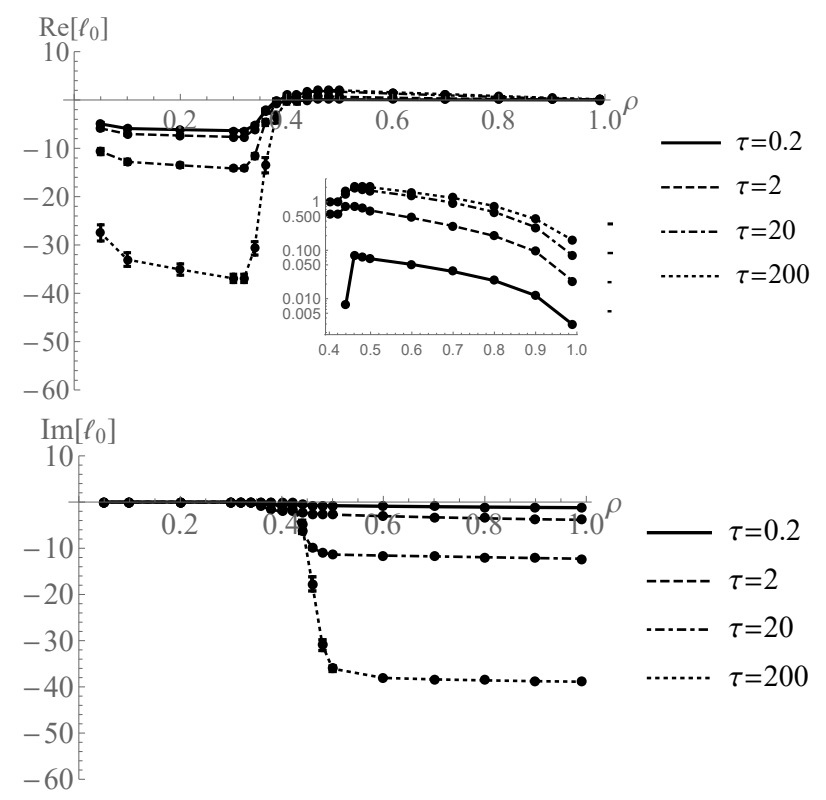

FIG. 5. Top: plot of the average value of the real part of the lowest (in real part) eigenvalue of the super-Laplacian $\Lambda^{\mathrm{AdLg}}$ for $N=40$ random regular $k_{i}=8$ with $\sigma=1, \Omega=2.5$, averaged over 300 instances, as a function of the density of controlled nodes $\rho$ and for different $\tau$; inset the real part on logarithmic scale; bottom: plot of the imaginary part of the eigenvalue.

\section{INSIGHTS FROM STABILITY ANALYSIS FOR VARYING CONNECTIVITY}

\section{A. Adaptive lag spectra for $k=8$ : towards an intuitive picture}

We now compute the spectra, specifically the lowest eigenvalue which informs the nature of stability of the two systems. Firstly, we draw attention to the property that the spectra for the adaptive lags model are complex. We show first the behaviour of the real and imaginary parts of the lowest eigenvalue for this model for $k=8$, as usual a characteristic example. Note that with our sign convention, a positive eigenvalue indicates stability of the fixed-point. In Fig. 5 we plot this lowest eigenvalue, $\ell_{0}$, as a function of the density of controlled nodes $\rho$, and for four choices of $\tau$. Here we average over 300 frequency and graph instances for the four values of $\tau$.

We observe a number of prominent features in Fig. 5: that for $\rho=1$ the real part of the eigenvalue becomes extremely small (emphasised by examining the logarithmic plot in the inset of Fig. 5) while its imaginary part attains its largest value; and that below a certain threshold of $\rho=0.3$ eigenvalues become negative real valued. The former result indicates long oscillatory transients in the dynamical approach to equilibrium while the latter result demonstrates instability of the dynamics below a critical density of controlled oscillators. Indeed, we ob- serve in full numerical solutions to the system at $\rho=1$ long term oscillations (as are visible in the $\rho=0.8$ case in Fig. 2) while for small $\rho$ the system seeks to synchronise to the mean frequency of the oscillator ensemble. Comparing these situations to full numerical solutions of the $N=40$ case we observe oscillations for $\rho=1$ even after $t=10,000$, where corresponding results for $0.7<\rho<0.9$ show consistent convergence to equilibrium at the driving frequency $\Omega=2.5$. Below $\rho=0.7$ the transients to external synchronisation become longer and beyond the range of the Mathematica calculations used here. We also see, as predicted, that increasing $\tau$ has a more significant impact on the imaginary part of the lowest eigenvalue - in the stable regime. In the unstable regime, larger $\tau$ has the effect of amplifying the negative real part. Note that the value of $\rho$ at which the instability occurs does not change - because this only depends on the $\alpha_{i}, \mu_{j}$ which are $\tau$ independent.

The origins of the instability may be understood by examining the values of $\alpha_{i}$ and $\mu_{i}$ across the range of $\rho$, as shown in Fig. 3. Note that as $\rho$ decreases, the size of $\mathcal{G}_{1}$ decreases while that of $\mathcal{G}_{2}$ increases; thus the number of $\mu_{i} \neq 0$ decreases (fewer lags are introduced) and the number of $\alpha_{i} \neq 0$ increases. In Fig. 3 we highlight in both cases the position of the value of $-\pi / 2$. It is evident that, for this size network, it is just below $\rho=0.4$ that the sum $\alpha_{i}+\mu_{j}$ exceeds $\pm \pi / 2$ where the cosine factor in the super-Laplacian terms, such as $\Lambda^{\vartheta \vartheta}$, changes sign. From the inset of Fig. 3 we see that the average value of $\cos \left(\mu_{i}+\alpha_{i}\right)$ indeed changes sign at $\rho=0.3$. When $\rho=0.3$ the $\mu_{i}$ achieve their largest average value - and this is where the instability is evident in the average eigenvalue. Because of this, the equilibria cease to be relevant below $\rho=0.3$. At a more intuitive level, varying $\rho$ means varying the population of tightly controlled oscillators in relation to the increasingly splayed uncontrolled oscillators. Instability is triggered once the splay of the latter population increases beyond a threshold where the combined phase lag and splay exceed $\pi / 2$.

This final observation on the $k=8$ example warrants a further comment. In previous experience with twocluster approaches $[36,37]$, we observed that the Lyapunov instability closely coincided with the point where the equations defining the equilibrium manifold, the analogues of Eqs. (10,11), failed to have static solutions. Thus, there, no instability on a static two-cluster solution was found. Here, even when increasing $\Omega$ we find that static solutions in many cases are obtainable - but the spectrum shows instability. This is simply a consequence of the fact that we now allow an arbitrary degree of splay. The instability might be said to correspond to where the oscillators of sub-graph $\mathcal{G}_{2}$ might no longer be called a single 'cluster'.

In summary then, we see that the spectral properties in the vicinity of the two cluster fixed point explain both the long transients in dynamics for $\rho \rightarrow 1$, and increasing $\tau$, as well as instability for lower values of $\rho$ where the splay of only frequency synchronised uncontrolled nodes 
overwhelm the dynamics.

\section{B. Comparing adaptive lag and reference models across values of $k$}

We have, till now, deferred discussion of the spectrum for the reference model other than to point out it is entirely real. We consider this now for various $k$, alongside plots of the real part of the lowest eigenvalue of the adaptive lag model in Fig. 6. Note that we cease to show the imaginary part here, for brevity, but its properties are analogous to the behaviour seen for $k=8$ in Fig. 5. In the first column we show results for the lowest eigenvalue for different $\eta$ of the reference model and in the second column the real part for different $\tau$ of the adaptive lag model, with the eigenvalue as a function of the density of controlled nodes, $\rho$. The value of $k$ increases down the columns.

For the reference model, we observe a clear transition as $\rho$ decreases, the eigenvalue correspondingly decreases until it reaches effectively zero. For $\eta=1$ the eigenvalue is extremely close to zero over the entire range of $\rho$. In some cases, at small $\rho$ a small negative value is observed but this appears to be a numerical instability in computing the spectrum. The point at which the eigenvalue effectively becomes zero varies for different connectivities $k$, with a trend to lower densities as $k$ increases once $\eta>1$.

To understand these behaviours we note that in the absence of the shift $\eta \cos \mu_{i}$ in $\Lambda^{\text {Ref }}$, the lowest eigenvalue will always vanish as is typical for graph Laplacians. Thus, while the $\mu_{i}$ are small at large $\rho$ (as in Fig. 4) the shift in the spectrum is positive. Once one of the $\mu_{i}$ cross the threshold of $-\pi / 2$ the shift is negative which can contribute to decreasing the otherwise positive spectral gap of the pure Laplacian. For $\eta=1$, the $\mu_{i}$ are already large at $\rho=1$, as is evident in Fig. 4. However at this point the combination $\eta \cos \mu_{i}$ is now less than one. As $\rho$ decreases, the number of oscillators resisting the Kuramoto interaction decreases and so the natural Kuramoto dynamics allow some degree of convergence in phases - and hence the $\mu_{i}$ decrease. Intuitively, at $\eta=1$, which equals the Kuramoto coupling $\sigma=1$ chosen here, the externally driven is a larger frustrated Kuramoto system with a wider frequency distribution. For $\eta<1$, not shown here, the shift $\eta \cos \mu_{i}$ in the spectrum assumes small non-zero values that fluctuate in sign around zero.

We conclude then that for $\eta>1$ there is a transition to marginal stability; for $k=4$ it occurs at $\rho \approx 0.5$, with decreasing density as $k$ increases so that at $k=14$ the critical density is as low as $\rho \approx 0.2$ for $\eta \sim 10$. Indeed we even see that this threshold value in $\rho$ varies with $\eta$ in the range $1<\eta<5$, a property seen in the numerical results of Fig. 1. In contrast to the adaptive lag model, the transition for the reference model is not to an instability but to marginal stability which would require a higher order analysis to establish definitively the Lyapunov proper- ties. Moreover, the marginal stability is consistent with the property that we could not reach equilibrium in the full numerical results for such low values of $k$.

Contrastingly, and turning to the second column of Fig. 6 at very low $k$ the adaptive model shows instability across all values of density of controlled oscillators and for any value of $\tau$. At $k=5$ the critical density appears at $\rho \approx 0.7$. At $k=8$ the adaptive model is competitive with the reference model insofar as the critical densities are approximately the same. But also, as we have already observed, there is little sensitivity to the value of $\tau$ across the densities allowing for stability. Also, the critical density for the external driving model shows greater sensitivity to $\eta$ than does the adaptive lag model with respect to $\tau$. At high connectivity, the external driving requires higher coupling, $\eta>5$. The adaptive lags, on the other hand, continue to show insensitivity to the value of $\tau$, with critical density $\rho \approx 0.3$.

All these results are consistent with the behaviours in Fig. 1, in the values of the critical densities where the systems may synchronise to the external frequency and the relative sensitivity of the reference model to $\eta$, and the insensitivity of the adaptive lag model to the value of $\tau$. Moreover, through the stability analysis, by identifying the role of splay with controlled and uncontrolled oscillators, we have gained insight into the mechanisms behind the relative performance of these control models.

\section{COUPLING DEPENDENCE}

We finally investigate the dependence of the two models on the coupling strength $\sigma$. To this end we fix a relatively low value of the density $\rho$ where both models show some sensitivity to $\tau$ and $\eta$ respectively, in this case $\rho=0.4$. Given its success thus far, we use the linearised theory and plot the lowest eigenvalue of the superLaplacian of the two models as a function of $\sigma$ for various $\tau$ and $\eta$ respectively. These are shown in Fig. 7. Given the greater sensitivity of the external driving model we include a broader range of $\eta$.

We observe for the adaptive lag model a clear transition from instability to stability as coupling is increased. However, the transition value of coupling is only weakly sensitive to the value of $\tau$ and once the transition has occured further increases in coupling does not achieve any further increase in the eigenvalue. We may thus speak of a critical coupling. Notable in this plot is that at the critical $\sigma$ the curves swap in value of $\tau$ : below the critical coupling large values of $\tau$ give larger negative eigenvalues compared to lower $\tau$ values. Therefore at low values of coupling, large values of $\tau$ increase instability arguably because of competition between phases strongly coupled to the adaptive lags and the (at $\rho=0.4$ ) larger population of weakly coupled oscillators. Intuitively, further increases in coupling will tighten the splay of phases that are not coupled to the lags but plays no part in improving the stability of the system, namely the decay rate of 

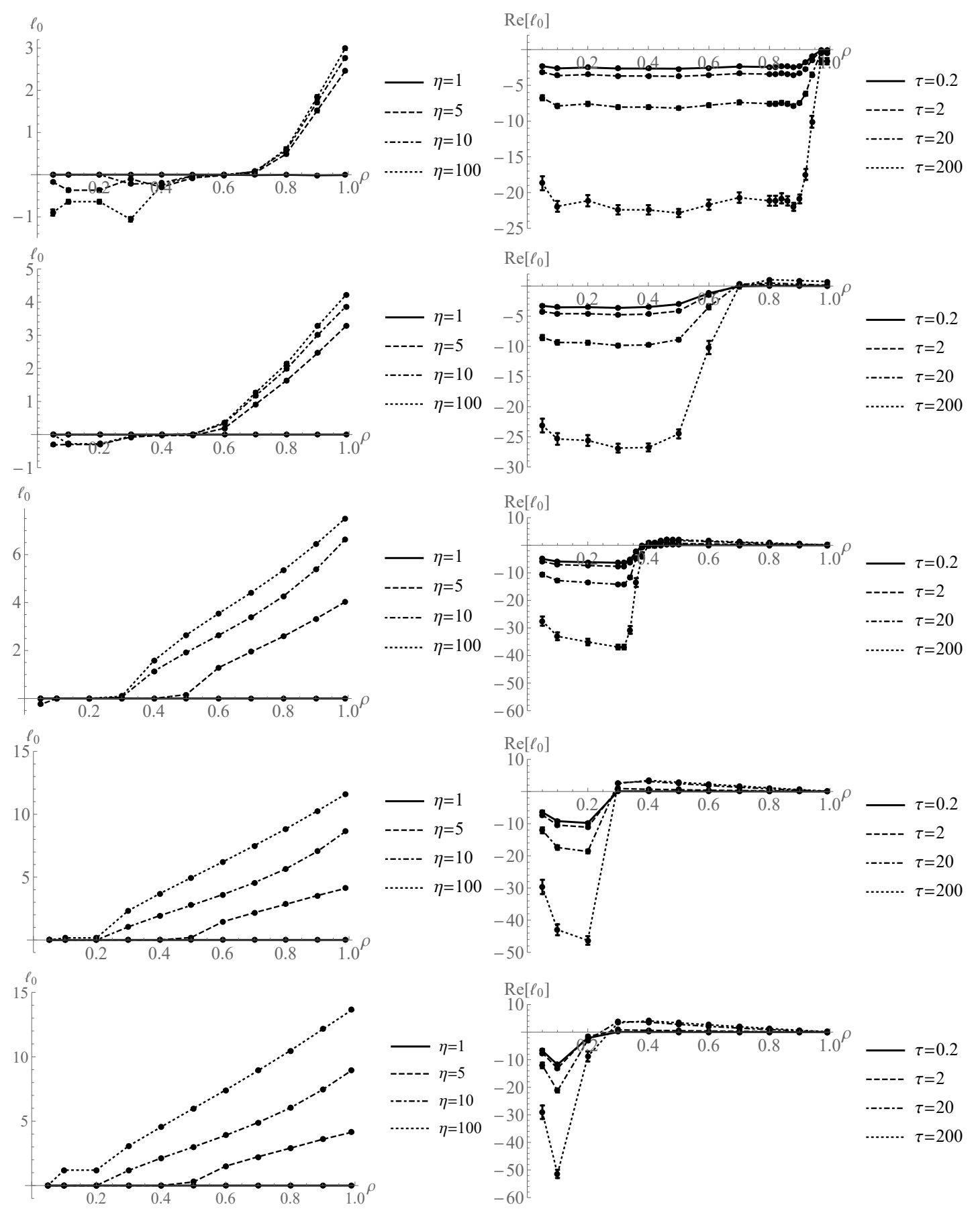

FIG. 6. Plots of the average value of the (real part of the) lowest eigenvalue of the super-Laplacian for $N=40$ random regular graphs with $\sigma=1, \Omega=2.5$ as a function of the density of controlled nodes $\rho$, left for the reference model $\Lambda^{\text {Ref }}$ for different $\eta$, and right for the adaptive lag model $\Lambda^{\mathrm{AdLg}}$ for different $\tau$, varying the connectivity as $k=4,5,8,12,14$ vertically down.

fluctuations from equilibrium. Contrastingly, for the external driving model there is no transition to instability as such. For $\eta=1$ there is marginal stability for any value of coupling. Beyond this point, essentially $\eta$ and $\sigma$ are interchangeable in terms of driving the system to synchronise: increasing both to any degree results in a more stable system with the two mechanisms operating in concert.

\section{CONCLUSIONS}

We have compared two models for control in the Kuramoto-Sakaguchi model, where control means both 


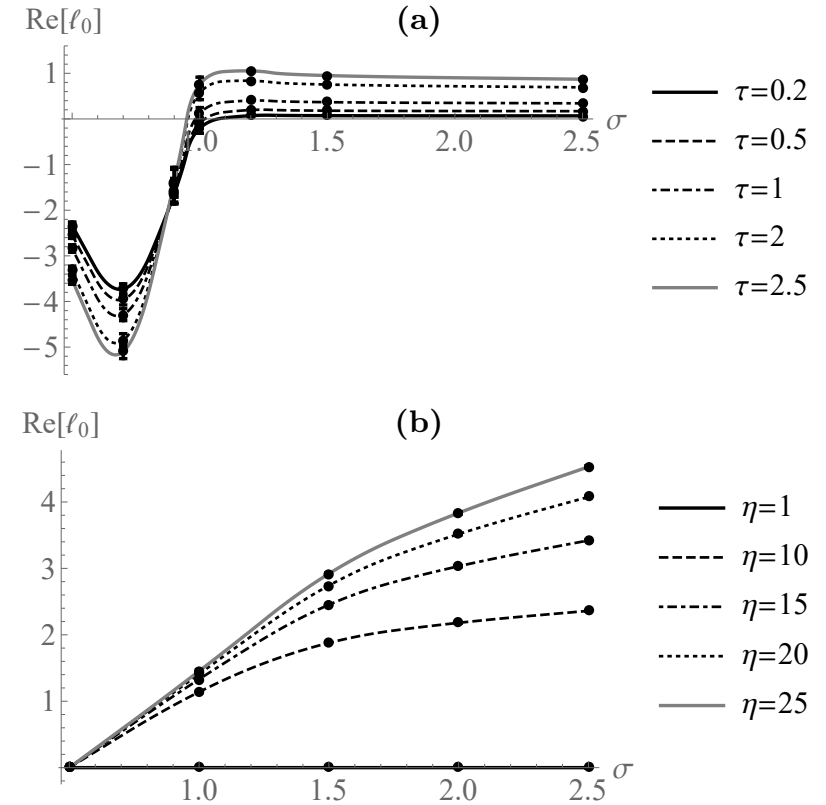

FIG. 7. (a): plot of the average value of the real part of the lowest (in real part) eigenvalue of the super-Laplacian $\Lambda^{\mathrm{AdLg}}$ for the adaptive lags for $N=40$ random regular $k_{i}=8$ with $\Omega=2.5, \rho=0.4$, averaged over 300 instances, as a function of the coupling strength $\sigma$ and for different $\tau$. (b): the average value of the real part of the lowest (in real part) eigenvalue of the external driven model, again for $N=40$ random regular $k_{i}=8$ with $\Omega=2.5, \rho=0.4$, averaged over 300 instances, as a function of the coupling strength $\sigma$ but for different $\eta$. Error bars in both cases are the size of the data points.

to drive more oscillators to participate in the synchronisation and to drive the collective frequencies to some externally chosen value. The adaptive lag model overall performs poorly compared to an external driving reference model for low connectivity. However, once connectivity is in excess of average degree $k=5$ the adaptive model is able to achieve synchronisation to the external frequency down to densities of controlled oscillators comparable to the reference model. Namely, higher synchro- nisation is achieved at collective frequencies other than the mean of the native frequency ensemble. The adaptive lag mechanism achieves the effect with at least as many control nodes on average but certainly with weaker coupling strengths required, down to an order of magnitude smaller couplings $\tau$ than for the reference model driving strength $\eta$. Indeed, examination of the coupling dependence of the adaptive lag system shows that beyond a critical coupling, further increases in $\sigma$ are not required to substantially change the stability properties of the system. The external driving model never shows a transition to instability but merely marginal stability for $\eta=1$ at all values of coupling. Remarkably, stability analysis of these two systems both predicts the behaviours seen in full numerical calculations, and also provides insight into the mechanisms for the two models.

We propose that the adaptive lag model may be applicable to achieve control in a range of engineered systems, such as autonomous/robotic control or energy distribution systems, as discussed in the introduction [14-17], specifically where the interactions between entities are mediated by technology. Just as the oscillators at the nodes of the network may represent certain technological agents or sub-systems, according to the application domain, so too one may envisage technology agents on the links of the network generating the lags in the system. The adaptive lag model therefore provides a co-evolution mechanism of these technological agents that serve to effectively regulate the overall dynamics of the system, however not through a top-down mechanism (as in the reference model), but through bottom-up processes. In this respect, the adaptive lag model promises a valuable approach to control through emergence in complex systems.

\section{ACKNOWLEDGMENTS}

One of us (ACK) was supported in this work by a Chief Defence Scientist Fellowship and expresses gratitude for the hospitality of the University of Southampton where much of this work was completed.
[1] Y. Kuramoto, Chemical Oscillations, Waves and Turbulence, Springer, Berlin, 1984

[2] J.A. Acebron et al., Rev.Mod.Phys. 77, 137, 2005

[3] S.N. Dorogovtsev, A.V. Goltsev, J.F.F. Mendes, Rev.Mod.Phys. 80, 1275, 2008

[4] A. Arenas et al., Phys.Rep. 469, 93, 2008

[5] Dörfler, F. Bullo, F. (2014). Synchronization in complex networks of phase oscillators: a survey, Automatica 50, 1539

[6] H. Hong, M.Y. Choi, B.J. Kim, Phys.Rev. E 65, 026139, 2002

[7] T. Ichinomiya, Phys.Rev. E 70, 026116, 2004

[8] J.G. Restrepo, E. Ott,, B.R. Hunt, Phys.Rev. E 75,
066106, 2007

[9] E. Oh, D.-S. Lee, B. Kahng, D. Kim, Phys.Rev. E, 011104, 2007

[10] J. Gómez-Gardenes, Y. Moreono, A. Arenas, Phys.Rev. E 75, 066106, 2007

[11] A.H. Dekker, J.Artif.Soc.Soc.Simul. 10, 6, 2007.

[12] M. Brede, Eur.Phys.J.B 62, 87, 2008

[13] R.F. Stengel, Optimal control and estimation, Dover, USA, 1994

[14] D.A. Paley, N.E. Leonard, R. Sepulchre, D. Grunbaum, J.K. Parrish, Oscilator models and collective motion, IEEE Control Systems Magazine 27 (4) 89-105, 2007

[15] F. Doerfler, F. Bullo, Synchronization and transient sta- 
bility in power networks and non-uniform Kuramoto oscillators, SIAM J. Control and Optimization 50 (3), 16161642,2012

[16] S. Aoi, K. Tsuchiya, Locomotion control of a biped robot using nonlinear oscillators, Autonomous robots 19 (3), 219-232, 2005

[17] T. Mizumoto et al., Human-robot ensemble between robot theremionist and human percussionist using coupled oscillator model, IEEE/RSJ Int. Conf. on Intelligent Robots and Systems, Taipei, Taiwan, 1957-1963, 2010

[18] Y. Liu, J. Slotine, A. Barabasi, Controllability of complex networks, Nature 473, 167, 2011

[19] X.D. Arsiwalla, Conference on Complex Systems, Amsterdam, 2016

[20] G. Li, W. Hu, G. Xiao, L. Deng, P. Tang, J. Pei, L. Shi, Minimum-cost control of complex networks, New J. Phys. 18, 013012, 2016

[21] M. Posfai, J. Gao, S.P. Cornelius, A.L. Barabasi, R. D'Souza, Controllability of multiplex, multi-time-scale networks, Phys.Rev.E 94, 032316, 2016

[22] O. Gjata, M. Asllani, L. Barletti, T. Carletti, Hamilton control of Kuramoto oscillators, arXiv:1610.01640

[23] V. Garcia-Morales, J. Pellicer, J.A. Manzanares, Ann.Phys. 323, 1844-1858, 2008

[24] A.C. Kalloniatis, Entropy and stability of phase synchronisation of oscillators on networks, Ann.Phys. 348, 127143,2014

[25] D. Witthaut, M. Timme, Kuramoto dynamics in Hamiltonian systems, Phys.Rev.E 90, 032917, 2014

[26] Y-H. Eom, S. Boccaletti, G. Caldarelli, Concurrent enhancement of percolation and synchronization in adaptive networks, Scientific Reports 6, 27111, 2016/doi:10.1038

[27] P.S. Skardal, D. Taylor, J.G. Restrepo, Complex macroscopic behavior in systems of phase oscillators with adaptive coupling, Physica D 267, 27-35, 2014

[28] F. Sorrentino, M. di Bernardo, F. Garofalo, G. Chen, Controllability of complex networks via pinning, Phys.Rev. E 75, 046103, 2007

[29] M. Porfiri, M. di Bernardo, Criteria for global pinningcontrollabiity of complex networks, Automatica 44, 31003106, 2008

[30] A. Clark, B. Alomair, L. Bushnell, R. Poovendran, Global practical synchronization in Kuramoto networks: a submodular optimization framework, arXiv:1411.5797v2, 2015

[31] P.S. Skardal, A. Arenas, Control of coupled oscillator networks with application to microgrid technologies, Sci.Adv.1:e1500339, 2015

[32] Y. Mozafari, A. Kiani, S. Hirche, Oscillator network synchronization by distributed control, IEEE Multiconference on systems and control MSC, 621-626, 2012

[33] M. Lohe, Synchronization control in networks with uniform and distributed phase lag, Automatica 54, 114-123, 2015

[34] P. Kundu, C. Hens, B. Barzel, P. Pal, Perfect synchronization in networks of phase-frustrated oscillators, arXiv:1801.05660v2, 2018

[35] M. Brede, A.C. Kalloniatis, Frustration tuning and perfect phase synchronization in the Kuramoto-Sakaguchi model, Phys.Rev.E 93, 062315, 2016

[36] A.C. Kalloniatis, M.L. Zuparic, Fixed points and stability in the two-network frustrated Kuramoto model, Physica A 447, 21-35, 2016
[37] A.B. Holder, M.L. Zuparic, A.C. Kalloniatis, Gaussian noise and the two-network frustrated Kuramoto model, Physica D 341, 10-32, 2017

[38] B. Bollobás, Modern Graph Theory, Graduate Texts in Mathematics, Springer, New York, 1998

[39] L.M. Pecora, T.L. Carroll, Phys. Rev. Lett. 80, 2109, 1998

[40] M. Fiedler, Czech. Math. J. 23 (98), 298, 1973

[41] A. Arenas, A. Diaz-Guilera, C.J. Pérez-Vincente, Phys.Rev.Lett. 96, 114102, 2006

[42] P.N. McGraw, M. Menzinger, Phys.Rev.E 77, 031102, 2008

[43] A.C. Kalloniatis, Phys.Rev.E 82, 066202, 2010

[44] B. Mohar, in Graph Symmetry: Algebraic Methods and Applications, Eds. G. Hahn, G. Sabidussi, NATO ASI Ser. C 497, Kluwer, 225-275, 1997 